\title{
A case of gadobenate dimeglumine-induced anaphylactic shock: a case report
}

\author{
Jiwei Huang", Zhuying Liu", Caiyun Jiang", Lejia Xu, Weifeng Zheng \\ Department of Pharmacy, Third Affiliated Hospital of Sun Yat-sen University, Guangzhou, China \\ \#These authors contributed equally to this work. \\ Correspondence to: Jiwei Huang. Department of Pharmacy, Third Affiliated Hospital of Sun Yat-sen University, Guangzhou, China. \\ Email: $947543512 @ q q . c o m$.
}

\begin{abstract}
A 70-year-old man was admitted to our hospital due to "liver cirrhosis; grade 3 hypertension; pulmonary infection”. On May 27, 2019, during upper abdomen plain and enhanced magnetic resonance imaging (MRI) and magnetic resonance cholangiopancreatography (MRCP), the patient experienced anaphylactic shock, manifested as sudden unconsciousness and lack of response, after intravenous administration of gadobenate dimeglumine $\left(\right.$ Multihance ${ }^{\circledR}$ ). Gadobenate dimeglumine is a paramagnetic contrast used during diagnostic MRI. It has hepatobiliary specificity with very good imaging performance. A small amount is absorbed by normal liver cells after intravenous injection and excreted via the bile ducts while maintaining the chemical structure of gadobenate dimeglumine. It allows the visualization of local angiogenesis and perfusion, which reflect the hepatic blood supply and recent liver function, thereby providing a reference for clinical diagnosis. Gadobenate dimeglumine intravenous injection may cause adverse reactions such as nausea, dizziness, and anaphylactic shock. Anaphylactic shock is a known serious adverse reaction of gadobenate dimeglumine injection. In this paper, we report a case of gadobenate dimeglumine-induced anaphylactic shock based on the temporal relationship between the onset of symptoms and the injection. The patient received chest compressions and balloon-assisted ventilation in addition to rehydration and volume expansion and vasoactive drugs to maintain blood pressure, etc. The patient died despite treatments. In the clinical, physicians, nurses, and clinical pharmacists should closely monitor patients and promptly discontinue drug administration and provide symptomatic care in case of adverse reactions.
\end{abstract}

Keywords: Magnetic resonance imaging (MRI); gadobenate dimeglumine; anaphylactic shock; case report

Submitted Mar 24, 2021. Accepted for publication Jun 03, 2021.

doi: 10.21037/apm-21-1076

View this article at: http://dx.doi.org/10.21037/apm-21-1076

\section{Introduction}

In modern medicine, precise diagnosis and treatment increasingly depends on imaging. As an imaging technique, magnetic resonance imaging (MRI) is advancing rapidly and has become widely used in recent years. Gadobenate dimeglumine is a commonly used contrast in enhanced MRI that helps clarify the number of lesions, identify lesions that may be missed on plain scans, distinguish tumors and surrounding edema, and facilitate qualitative diagnosis. Gadobenate dimeglumine is clinically indicated for the differential diagnosis of liver cancer and cholangiocarcinoma (1-4), but attention must be paid to its safety as it has been reported to induce anaphylactic shock (5). In this paper, we will report a case of gadobenate dimeglumine-induced anaphylactic shock. Unfortunately, the patient, who had cirrhosis, died despite treatment. Healthcare professionals should closely monitor patients for serious adverse reactions associated with gadolinium contrast agents.

We present the following article in accordance with the CARE reporting checklist (available at http://dx.doi. org/10.21037/apm-21-1076). 


\section{Case presentation}

A 70-year-old man visited the local hospital in April 2019 for unexplained fatigue and cough. He had no chest tightness, shortness of breath, chest pain, heart palpitations, skin or sclera jaundice, anorexia, abdominal distension, vomiting of blood, or black stools. Laboratory tests showed white blood cells (WBCs) $4.24 \times 10^{9} / \mathrm{L}$, hemoglobin $115 \mathrm{~g} / \mathrm{L}$, platelets $53 \times 10^{9} / \mathrm{L}$, total bilirubin $36.7 \mu \mathrm{mol} / \mathrm{L}$, albumin $32.9 \mathrm{~g} / \mathrm{L}$, alanine aminotransferase $31 \mathrm{U} / \mathrm{L}$, aspartate aminotransferase $51 \mathrm{U} / \mathrm{L}$, and prothrombin time (PT) 14.9 s. Computed tomography (CT) showed liver cirrhosis and splenomegaly. His symptoms improved after liver-supporting and jaundice-reducing care. On May 15, 2019, the patient was admitted to the Department of Infectious diseases at our hospital for "liver cirrhosis; grade 3 hypertension; pulmonary infection". He had a history of hepatitis $\mathrm{E}$ with successful treatment and a history of chronic hypertension. In April 2019, he was considered to have type 2 diabetes as laboratory tests performed at the local hospital showed elevated blood glucose. He had no known history of infectious diseases, such as tuberculosis, hepatic distomiasis, or schistosomiasis; chronic diseases, such as coronary heart disease or kidney disease; blood transfusion or blood transfusion reactions; major trauma and surgery; or allergies to food or drugs. A physical examination performed at admission showed that he was consciousness and able to appropriately respond to questions, with normal orientation and numeration ability. He had no pale or yellow complexion. Additional exams showed palmar erythema (+), spider nevi (-), chest telangiectasias $(+)$, no yellow skin, no skin ecchymosis or hyperpigmentation, no sclera jaundice, no bulbar conjunctiva edema, no pale eyelid conjunctiva, normal heart and lungs, smooth abdomen, no visible abdominal veins, normal umbilicus, soft abdominal muscles, tenderness (-), rebound tenderness (-), no palpable mass, Murphy's sign (-), liver not palpable below the right costal arch, spleen not palpable below the left costal arch, percussion tenderness over the liver area (-), shifting dullness (-), normal bowel sounds, no pitting edema in the lower limbs, and flapping tremor (-). Laboratory tests showed WBC $2.25 \times 10^{9} / \mathrm{L}$, hemoglobin $114 \mathrm{~g} / \mathrm{L}$, platelets $55 \times 109 / \mathrm{L}$, total bilirubin $36.7 \mu \mathrm{mol} / \mathrm{L}$, albumin $32.9 \mathrm{~g} / \mathrm{L}$, alanine aminotransferase $37 \mathrm{U} / \mathrm{L}$, aspartate aminotransferase $71 \mathrm{U} / \mathrm{L}$, PT $13.8 \mathrm{~s}$, glycosylated hemoglobin $5.2 \%$, creatinine $64 \mu \mathrm{mol} / \mathrm{L}$, hepatitis B surface antigen ( $\mathrm{HBsAg})(-)$, hepatitis B surface antibody (anti-HBs) (+), hepatitis B e antigen (HBeAg)
(-), hepatitis B e antibody (anti-HBe) (+), and hepatitis B core antibody (anti-HBc) (+). After admission, the patient received symptomatic and supportive care, including liversupporting therapy, albumin infusion, cough-reducing medicine and expectorant, and hypotensive drugs. The cause of liver cirrhosis was unknown, and bile duct disease was considered. Therefore, the patient was scheduled to undergo upper abdomen MRI and magnetic resonance cholangiopancreatography (MRCP) to rule out bile duct conditions. On the afternoon of May 27, 2019, the patient went to the Department of Radiology for plain and enhanced upper abdomen MRI and MRCP. Before the MRI, the patient took oral gadobenate dimeglumine solution (3 mL diluted in $500 \mathrm{~mL}$ of normal saline) and received an intramuscular injection of anisodamine $(10 \mathrm{mg})$. The MRI started at 7:00 pm. At 7:35 pm, $5.8 \mathrm{~mL}$ of gadobenate dimeglumine solution (Multihance, lot: 1810005B, Shanghai Bracco Sine Pharmaceutical Co., Ltd., China) and $20 \mathrm{~mL}$ of sodium chloride solution were bolus injected (iv) via a high-pressure injector. A few minutes later, the patient felt unwell during the two-phase dynamic enhanced scan. The physician immediately entered the exam room to communicate with the patient. The patient complained of nausea and was uncooperative. The scan was terminated. With the assistance of staff, the patient walked to the entrance of the scan room and then suddenly lost consciousness and was unresponsive. Gadobenate dimeglumine-induced anaphylactic shock was diagnosed based on the temporal relationship between the onset of symptoms and the injection. The medical staff immediately established vein access for rehydration and volume expansion and administered oxygen therapy. In addition, cardiopulmonary resuscitation was promptly performed due to the loss of major arterial pulse and respiration, and drugs including epinephrine $1 \mathrm{mg}$, dexamethasone $5 \mathrm{mg}$, and methylprednisolone $80 \mathrm{mg}$ were intravenously injected. At 7:48 pm, the patient's blood pressure was 105/41 $\mathrm{mmHg}$, and his pulse was $125 \mathrm{bpm}$. The emergency department was contacted for help. Upon arrival, the emergency physician found the patient lying supine on the floor, with a blue or purple complexion, no responsiveness, and no carotid arterial pulse. Chest compression and balloon-assisted ventilation continued. The patient's heart sounds resumed, and he was transferred to the emergency room for further treatment. Cardiac arrest recurred during transfer, and rescue therapy, including chest compressions and balloonassisted ventilation, was readministered. In the emergency room, the patient continued to receive chest compressions 
A 70-year-old man was admitted to the Department of Infectious Diseases at our hospital for "liver cirrhosis; hypertension grade 3 ; pulmonary infection."

\begin{abstract}
Upper abdomen MRI and MRCP were indicated to rule out bile duct disease, and the patient took oral gadobenate dimeglumine solution and received an intravenous injection of gadobenate dimeglumine in preparation for these examinations. During the examination, the patient felt unwell; he complained of nausea and was uncooperative. The exam was terminated. Later, the patient suddenly lost consciousness and became unresponsive. Despite active treatments, the patient remained unconscious. The family was notified of the patient's critical condition and adverse prognosis. The patient was transferred to the $\mathrm{CCU}$ for further treatment.
\end{abstract}

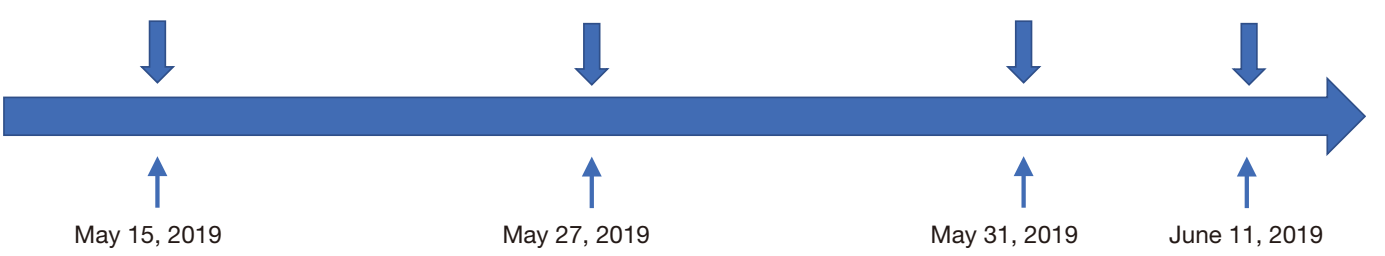

Figure 1 Timeline.

and balloon-assisted ventilation in addition to rehydration and volume expansion [compound ammonium chloride injection $(500 \mathrm{~mL}$, iv), $5 \%$ glucose injection $(500 \mathrm{~mL}$, iv)] and vasoactive drugs [norepinephrine (18 $\mathrm{mg}$, bolus) + dopamine (180 mg, bolus)] to maintain blood pressure. An anesthesiologist was consulted and performed emergency endotracheal intubation, and the patient was connected on a ventilator. Additionally, a cardiologist was consulted and performed emergency placement of a temporary pacemaker. Despite active treatments, the patient remained unconscious. Norepinephrine $(18 \mathrm{mg}$, iv) and dopamine (180 mg, iv) were administered to maintain blood pressure. Physical examination showed blood pressure 90/45 mmHg and heart rate $103 \mathrm{bpm}$. Blood oxygen saturation (SpO2) was $100 \%$ with ventilator-assisted ventilation. The pupils were round and of equal size (approximately $6 \mathrm{~mm}$ ), with no light reflex. The carotid arterial pulse was palpable. The family was notified of the patient's critical condition and adverse prognosis. The patient was transferred to the critical care unit (CCU) for further treatment. Unfortunately, he underwent brain death on May 31 and clinical death on June 11 due to his rapidly progressing and uncontrolled conditions. The timeline is shown in Figure 1.

Written informed consent was obtained from the patient for publication of this manuscript and any accompanying images. The content involved here was following the ethical standards of the institutional and/or national research committee(s) and with the Helsinki Declaration (as revised in 2013).

\section{Discussion}

The causes of liver cirrhosis are various, which can be roughly divided into infectious, chemical damage, autoimmune, cholestasis, metabolic and genetic, vascular. Cholestasis includes congenital biliary atresia, allagile syndrome, progressive familial cholestasis and so on. Clinically, there are many examination methods for bile duct diseases, including contrast-enhanced ultrasound, two-dimensional ultrasound, computed tomography, magnetic resonance imaging, magnetic resonance cholangiopancreatography, probe confocal laser micro endoscopy and so on. The cause of liver cirrhosis was unknown, and bile duct disease was considered. Therefore, the patient was scheduled to undergo upper abdomen MRI and magnetic resonance cholangiopancreatography (MRCP) to rule out bile duct conditions.

Gadobenate dimeglumine is a paramagnetic contrast used during diagnostic MRI. It has hepatobiliary specificity $(6,7)$ with very good imaging performance. A small amount is absorbed by normal liver cells after intravenous injection and excreted via the bile ducts while maintaining the chemical structure of gadobenate dimeglumine. It allows the visualization of local angiogenesis and perfusion, which reflect the hepatic blood supply and recent liver function, thereby providing a reference for clinical diagnosis (2). Gadobenate dimeglumine-enhanced dynamic MRI effectively improves the diagnostic accuracy for focal liver 
lesions (8). Studies $(9,10)$ have shown that during MRI, gadobenate dimeglumine enhancement of the hepatobiliary phase effectively helps identify focal nodular hyperplasia, atypical nodular hyperplasia cirrhosis, and liver cancer. The recommended dose of gadobenate dimeglumine is $0.1 \mathrm{mmol} / \mathrm{kg}$ body weight in adults $(0.2 \mathrm{~mL} / \mathrm{kg}$ for $0.5 \mathrm{M}$ solution). There is no need to adjust the dose in patients with liver function damage. Therefore, the dose used for this patient was within the recommended range.

Contrast-related allergic reactions vary greatly in severity, and they can be divided into acute, late, or ultra-late adverse reactions based on onset time. Gadolinium-containing contrast agents are widely used during MRI and have fewer but possibly more severe adverse reactions than iodinebased contrast agents. As with other gadolinium contrast agents, gadobenate dimeglumine-induced allergies/allergic/ hypersensitivity reactions have been reported (11). Their severity varies, and they may be serious or life-threatening and can lead to anaphylactic shock (12) or even death. Adverse reactions may involve one or more organs and systems, including respiratory, cardiovascular, and/or skin and mucosal tissues (13), especially in patients with a history of asthma or other allergic diseases. Studies have shown that the overall incidence of gadobenate dimeglumine-induced adverse reactions is only $0.76 \%$ (14), which is lower than that of other MRI contrast agents. In a prospective observational safety study, more than 130,000 patients were followed up over 7.5 years for gadobenate dimeglumine-induced adverse events. The results showed that the incidence of acute adverse reactions was $0.18 \%$ (15), indicating that gadobenate dimeglumine has a good safety profile.

Patient's sex, age, site, and specific MRI procedure are related to acute adverse reactions (16). Anaphylactic shock is a known serious adverse reaction of gadobenate dimeglumine injection. In this patient, anaphylactic shock was probably related to gadobenate dimeglumine injection given the temporal relationship and known drug safety information. The Patient had known risk factors (age $>60$ years, grade 3 hypertension, and diabetes) for contrast agents. Further research is needed to investigate whether these factors contribute to anaphylactic shock.

Gadobenate dimeglumine cannot be mixed with other drugs for injection. Before gadobenate dimeglumine injection, the patient in the present study took oral gadobenate dimeglumine solution (diluted) and intramuscular anisodamine injection $10 \mathrm{mg}$. The purpose of oral gadobenate dimeglumine solution (diluted) is to suppress surrounding intestinal fluid signals and ensure good duodenum filling and quality images. Anisodamine is an $M$ receptor blocker. It can block the binding of acetylcholine and $M$ receptor, relax the smooth muscle of biliary tract, slow down peristalsis, reduce the artifacts caused by the movement of biliary tract, and maintain the clarity of scanning image, which is conducive to diagnosis. The main adverse reactions of anisodamine were accelerated heart rate, dysuria, dry mouth, blush and so on. No allergic reaction was reported. Anisodamine also has not been reported to increase the incidence of gadolinium meglumine allergic reactions (17). He was the only patient who experienced gadobenate dimeglumine-induced adverse reactions on the day of his examination. A review of 5-year data showed that the incidence of gadobenate dimeglumine-induced serious adverse reactions at our hospital is less than $0.01 \%$. Before using gadolinium meglumine, the patients were treated with liver protection, albumin supplement, cough and expectorant, and hypotension, and the basic condition was good. According to the correlation between the shock signs and the injection time, the patient was diagnosed as one case of anaphylactic shock caused by gadolinium meglumine, which involved multiple organ systems (respiratory, cardiovascular and liver). After treatment, the patient failed and eventually died. The patient's family has consented to the publication of this case report.

Diagnostic contrast agents (such as gadobenate dimeglumine) should be used in hospitals with emergency rescue equipment, such as cardiopulmonary resuscitation equipment, and trained medical staff. Before gadobenate dimeglumine injection, it is important to ask the patient's allergy history carefully and inform the patient of possible adverse reactions. The trained physicians control the injection pressure and speed of contrast agent and confirm that emergency rescue drugs are available to treat any adverse reactions (such as anaphylactic shock) that occur during the exam. After injection, the patient should be closely monitored for 15 minutes to ask whether they feel uncomfortable and make preparations for first aid. Because most of the serious adverse reactions occurred in this period of time. Moreover, the patient should be observed at the hospital for one hour after injection. At the end of the examination, patients need to be told to drink more water to promote drug excretion from the kidney. Once the patients have adverse reactions after medication, they must be treated symptomatically in time.

Clinicians should carefully weigh the clinical benefits and risks of gadobenate dimeglumine. The manufacturer's instructions for use and the China Food and Drug 
Administration (CFDA) approved package insert should be updated in a timely manner to reflect new safety information and reported risks in order to provide the best drug information to clinicians.

\section{Acknowledgments}

Funding: None.

\section{Footnote}

Reporting Checklist: The authors have completed the CARE reporting checklist. Available at http://dx.doi.org/10.21037/ apm-21-1076

Conflicts of Interest: All authors have completed the ICMJE uniform disclosure form (available at http://dx.doi. org/10.21037/apm-21-1076). The authors have no conflicts of interest to declare.

Ethical Statement: The authors are accountable for all aspects of the work in ensuring that questions related to the accuracy or integrity of any part of the work are appropriately investigated and resolved. Written informed consent was obtained from the patient for publication of this manuscript and any accompanying images. All procedures performed in this study involving human participants were in accordance with the ethical standards of the institutional and/or national research committee(s) and the Declaration of Helsinki (as revised in 2013).

Open Access Statement: This is an Open Access article distributed in accordance with the Creative Commons Attribution-NonCommercial-NoDerivs 4.0 International License (CC BY-NC-ND 4.0), which permits the noncommercial replication and distribution of the article with the strict proviso that no changes or edits are made and the original work is properly cited (including links to both the formal publication through the relevant DOI and the license). See: https://creativecommons.org/licenses/by-nc-nd/4.0/.

\section{References}

1. De Gaetano AM, Catalano M, Pompili M, et al. Critical analysis of major and ancillary features of LI-RADS v2018 in the differentiation of small $(\leq 2 \mathrm{~cm})$ hepatocellular carcinoma from dysplastic nodules with gadobenate dimeglumine-enhanced magnetic resonance imaging. Eur
Rev Med Pharmacol Sci 2019;23:7786-801.

2. Kirchner J, Sawicki LM, Deuschl C, et al. 18 F-FDG $\mathrm{PET} / \mathrm{MR}$ imaging in patients with suspected liver lesions: Value of liver-specific contrast agent Gadobenate dimeglumine. PLoS One 2017;12:e0180349.

3. Dioguardi Burgio M, Picone D, Cabibbo G, et al. MRimaging features of hepatocellular carcinoma capsule appearance in cirrhotic liver: comparison of gadoxetic acid and gadobenate dimeglumine. Abdom Radiol (NY) 2016;41:1546-54.

4. Xu CC, Tang YF, Ruan XZ. The value of Gd-BOPTAenhanced MRIs and DWI in the diagnosis of intrahepatic mass-forming cholangiocarcinoma. Neoplasma 2017;64:945-53.

5. Virtos M, Ruiz S, Mokrane FZ, et al. Anaphylactic reaction and cardiac arrest due to gadobenate dimeglumine. Anaesth Crit Care Pain Med 2015;34:247-8.

6. Schuhmann-Giampieri G, Schmitt-Willich H, Press WR, et al. Preclinical evaluation of Gd-EOB-DTPA as a contrast agent in MR imaging of the hepatobiliary system. Radiology 1992;183:59-64.

7. Hamm B, Staks T, Mühler A, et al. Phase I clinical evaluation of Gd-EOB-DTPA as a hepatobiliary contrast agent: safety, pharmacokinetics, and MR imaging. Radiology 1995;195:785-92.

8. Zhang D, Xu A. Application of dual-source CT perfusion imaging and MR I for the diagnosis of primary liver cancer. Oncol Lett 2017;14:5753-8.

9. Li XM, Chen Z, Xiao EH, et al. Diagnostic value of gadobenate dimeglumine-enhanced hepatocyte-phase magnetic resonance imaging in evaluating hepatic fibrosis and hepatitis. World J Gastroenterol 2017;23:3133-41.

10. Grazioli L, Morana G, Kirchin MA, et al. Accurate differentiation of focal nodular hyperplasia from hepatic adenoma at gadobenate dimeglumine-enhanced MR imaging: prospective study. Radiology 2005;236:166-77.

11. Raisch DW, Garg V, Arabyat R, et al. Anaphylaxis associated with gadolinium-based contrast agents: data from the Food and Drug Administration's adverse event reporting system and review of case reports in the literature. Expert Opin Drug Saf 2014;13:15.

12. Biagioni E, Coloretti I, Disalvo F, et al. Case report of a patient who survived after cardiac arrest and cardiogenic shock by anaphylactic reaction to gadolinium during magnetic resonance imaging. Radiol Case Rep 2020;15:266-8.

13. Virtos M, Ruiz S, Mokreane F, et al. Anaphylactic reaction and cardiac arrest due to gadobenate dimeglumine. 
Anaesth Crit Care Pain Med 2015;34:247-8.

14. Bleicher AG, Kanal E. Assessment of adverse reaction rates to a newly approved MRI contrast agent: review of 23,553 administrations of gadobenate dimeglumine. AJR Am J Roentgenol 2008;191:W307-11.

15. Fakhran S, Alhilali L, Kale H, et al. Assessment of Rates of Acute Adverse Reactions to Gadobenate dimeglumine Review of More Than 130,000 Administrations in 7.5 Years. AJR Am J Roentgenol
2015;204:703-6.

16. McDonald JS, Hunt CH, Kolbe AB, et al. Acute Adverse Events Following Gadolinium-based Contrast Agent Administration: A Single-Center Retrospective Study of 281945 Injections. Radiology 2019;292:620-7.

17. He B, Gong S, Hu C. Obscure gastrointestinal bleeding: diagnostic performance of 64-section multiphase CT enterography and CT angiography compared with capsule endoscopy. Br J Radiol 2014;87:20140229.

Cite this article as: Huang J, Liu Z, Jiang C, Xu L, Zheng W. A case of gadobenate dimeglumine-induced anaphylactic shock: a case report. Ann Palliat Med 2021;10(6):7126-7131. doi: 10.21037/apm-21-1076 